\title{
Power wheelchair navigation assistance using wearable vibrotactile haptics
}

\author{
Louise Devigne, Marco Aggravi, Morgane Bivaud, Nathan Balix, Catalin Stefan Teodorescu, \\ Tom Carlson, Tom Spreters, Claudio Pacchierotti, Marie Babel
}

\begin{abstract}
People with severe disabilities often rely on power wheelchairs for moving around. However, if their driving abilities are affected by their condition, driving a power wheelchair can become very dangerous, both for themselves and the surrounding environment. This paper proposes the use of wearable vibrotactile haptics for wheelchair navigation assistance. We use one or two haptic armbands, each composed of four evenly-spaced vibrotactile actuators, for providing different navigation information to power wheelchair users. With respect to other available solutions, our approach provides rich navigation information while always leaving the patient in control of the wheelchair motion. Moreover, our armbands can be easily adapted for different limbs and can be used by all those patients who are unable to safely maneuver a kinesthetic interface. The results of two human subjects studies show the viability and effectiveness of the proposed technique with respect to not providing any environmental cue. Collisions were reduced by $49 \%$ when using the vibrotactile armbands. Moreover, most subjects expressed a preference for receiving haptic feedback and found the armbands comfortable to wear and use.
\end{abstract}

\section{INTRODUCTION}

Power wheelchairs are used to overcome mobility impairments resulting from orthopaedic or neurological disabilities. Unfortunately, not everyone in need of a power wheelchair can use it. Some people with cognitive and/or visual impairments are in fact not able to safely drive a power wheelchair [1]. Navigation assistance can be of great help in these situations, enabling a larger set of disabled people to drive wheelchairs. This can be enforced by directly controlling the wheelchair in assist-as-needed (shared control) or autonomous ways [2], [3]. However, it is proven empowering to do a task yourself rather than having a machine do it for you [4], [5]. Moreover, users are sometimes reluctant to relinquish control and might feel uncomfortable driving a (semi-)autonomous machine. It is then often considered beneficial to leave the patient in total control of the driving.

In a rehabilitation context, users are known to greatly benefit from an enhanced understanding of the surrounding environment while driving [6]. For these reasons, leaving patients in total control of the wheelchair while providing them with rich navigation feedback is a promising technique. As visual and auditory feedback modalities are likely to distract the driver as they rely on already overstimulated sensory channels [7], using distributed haptic stimulation seems a promising approach [8]. Haptic feedback has been indeed proven to be more effective than visual or auditory feedback to warn car drivers about safety-related events [9]. For example, wearable vibrotactile stimulation has been successfully employed for providing navigation and environmental information in a variety of applications, using vibrotactile vests [10], rings [11], belts [12], and armbands [13], [14], [15]. In these applications, the navigation path can be defined by a direction or a set of waypoints to be reached, expressed w.r.t the user's heading or some limb position. Vibrotactile feedback is then

This work has been funded by the Inria associated team "ISI4NAVE" and has been carried out as part of the INTERREG VA FMA project "Assistive Devices for empowering disAbled People through robotic Technologies (ADAPT)".

L. Devigne, M. Bivaud, N. Balix, T. Spreters, and M. Babel are with INSA, Univ Rennes, CNRS, Inria, IRISA - Rennes, France. \{louise.devigne, morgane.bivaud, nathan.balix, tom.spreters $\} @$ insa-rennes.fr, marie.babel@irisa.fr

M. Aggravi and C. Pacchierotti are with CNRS, Univ Rennes, Inria, IRISA - Rennes, France. \{marco.aggravi, claudio.pacchierotti\}@irisa.fr

S. Teodorescu and T. Carlson are with the Aspire Centre for Rehabilitation Engineering and Assistive Techn., University College London - London, UK. \{t.carlson, s.teodorescu\}@ucl.ac.uk

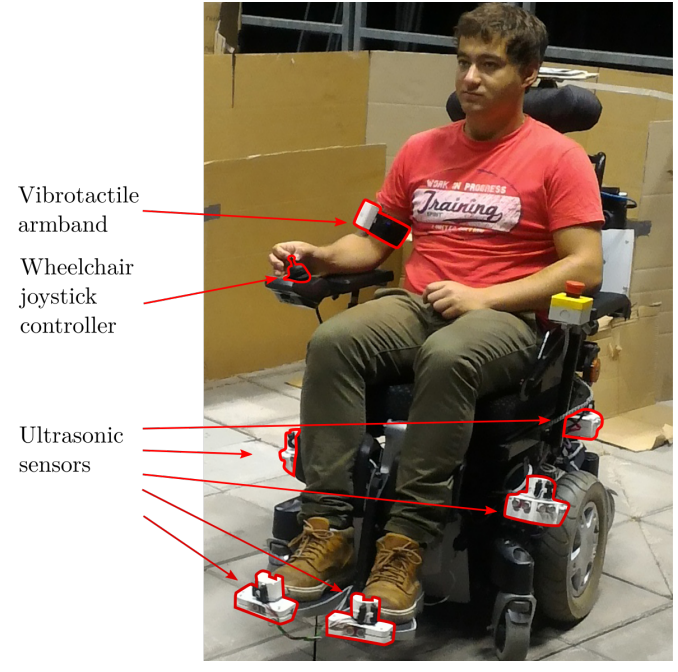

Fig. 1. General setup. A participant drives our power wheelchair along the circuit while being equipped with one vibrotactile armband. The wheelchair is commanded using a standard 2D joystick placed in the right armrest, and it is instrumented with 12 ultrasonic sensors to detect obstacles. The armband provides information either on a trajectory to follow or the presence of obstacles, depending on the condition being tested.

provided using either an attractive (move toward the vibration) or repulsive (move away from the vibration) approach. In the case of car driving, haptic feedback can be distinguished between 2 categories: guidance systems which mainly use continuous force feedback and warning systems which mainly use discrete event-based vibrations to communicate with the driver [16].

However, although promising, wearable vibrotactile stimulation has never been employed to aid wheelchair navigation. In fact, most studies investigating haptic feedback on wheelchairs use hapticenabled wheelchair controllers (i.e., kinesthetic joysticks). Some of these solutions help the user follow a predefined path [17], [18], [19], while others notify the presence of possibly dangerous obstacles [20], [21]. For example, Marchal-Crespo et al. [17] developed a robotic wheelchair trainer that guides users along a line drawn on the floor, with kinesthetic cues provided by a force-feedback joystick. Results show that the movements of the joystick, coupled with the haptic feedback, improved the steering ability of children without motor impairment as well as of one child with a severe motor impairment Sahnoun and Bourhis [22] used a force-feedback joystick to suggest obstacle-free directions while driving a power wheelchair. This system was tested in simulation on six healthy subjects, showing significant improvements w.r.t. providing no cues. More recently, Kucukyilmaz and Demiris [19] presented machine learning shared control policies from demonstrations offered by a human assistant. They trained a Gaussian process regression model to continuously regulate the level of assistance between the user and the robot, given the user's previous and current actions and the state of the environment. Results showed that the jerkiness of the user's joystick movements dropped.

These approaches have been proven to significantly decrease the number of collisions with the environment, making the driving much safer. Moreover, as the wheelchair already has a controller, providing 


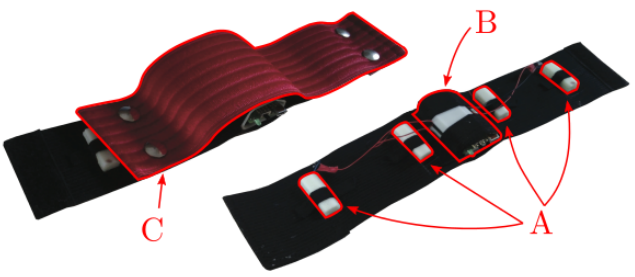

Fig. 2. Wearable vibrotactile armband. Each armband is composed of four vibrating motors (A), its electronics is enclosed in a 3D-printed case (B), and it can be covered by an elastic colored band (C).

haptic feedback through the same device minimizes the required modifications and their encumbrance. Nonetheless, such kinesthetic approaches have significant drawbacks. First, kinesthetic feedback does not really leave much choice to the driver, who is forced to follow the navigation guidance. Second, adding kinesthetic feedback to the wheelchair joystick can be quite expensive, given the cost of current grounded kinesthetic technologies. Third, providing kineshetic feedback on the same device controlling the motion of the wheelchair might lead to instability issues [23]. Finally, people in need of a power wheelchair often show upper limb weakness or reduced mobility, a condition that can make difficult to maneuver a kinesthetic controller. In this respect, the only non-kinesthetic solution has been presented by Yoon et al. [24], where skin stretch feedback was used either alone or combined with kinesthetic feedback provided by a grounded interface. Results show that control performance significantly improved when the combined skin-stretch/kinesthetic feedback was applied. However, no effect of skin stretch alone was shown. Although very promising, experiments were carried out in a fully-simulated environment, without actually moving or using a real power wheelchair.

In this paper, we propose the use of wearable vibrotactile haptic interfaces for providing navigation guidance to power wheelchair users. It directly addresses all the limitations of current navigation systems discussed above. Using one or two vibrotactile armbands, we can provide rich guidance feedback while leaving the user free to follow or deviate from the suggested path. Moreover, the armbands are inexpensive, easy to use, and very flexible. They can be worn either on the wrist, upper arm, or legs, depending on the preference and specific condition of the patient. They are also compatible with any wheelchair control system, including but not limited to the joystick controllers of commercially-available power wheelchairs. Each armband is composed of four evenly-spaced vibrotactile actuators, powered by a Li-ion battery and controlled by an embedded wireless electronic board Drivers receive information regarding the trajectory to follow or the presence of obstacles via vibrotactile stimuli, but they are always the ones in charge of controlling the motion of the wheelchair. Decoupling the feedback and control makes the teleoperation loop intrinsically safe [23]. We evaluate different feedback approaches in two real-world scenarios, where fifteen participants on a power wheelchair navigate two circuits of increasing difficulty.

\section{EXPERIMENTAL APPARATUS}

As therapists usually recommend 6-wheeled wheelchairs to people with driving difficulties [25], we use a Quickie Salsa $\mathbf{M}^{2}$ power wheelchair by Sunrise Medical. Note that the proposed approach is compatible with any power wheelchair. This wheelchair is controlled with a standard 2D joystick controller mounted on the right armrest. We instrumented the wheelchair with 12 SRF08 ultrasonic sensors attached on 3D-printed boxes placed at each corner of the wheelchair. Distance measurement was performed at $10 \mathrm{~Hz}$ with sensors sequentially triggered to ensure no interference.

Haptic feedback is provided by one or two vibrotactile armbands, depending on the experiment condition. Each armband is composed of

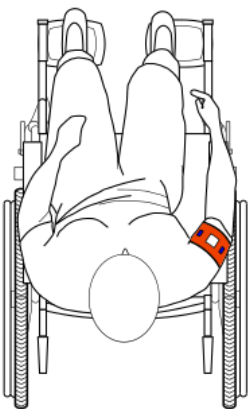

(a) Armband positioned one the right upper arm.

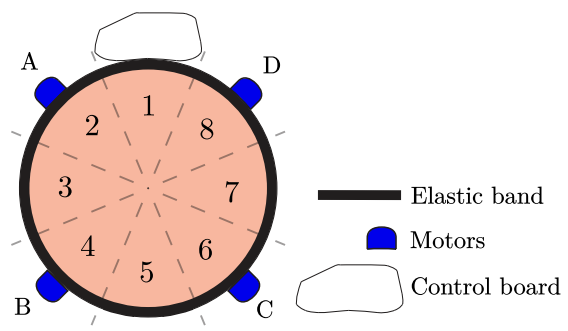

(b) Eight discretized directions, provided by the joint action of four vibrotactile motors.
Fig. 3. Experiment \#1: setup. Users wear one vibrotactile armband on their right upper arm. The armband provides information either on a trajectory to follow or the presence of obstacles, in the form of a direction with respect to the current wheelchair orientation.

four vibrotactile motors (Pico-Vibe 304-116, Precision Microdrives) attached to an elastic fabric strap. Each motor has an effective vibration frequency range between 80 and $280 \mathrm{~Hz}$, with an proportionallyrelated amplitude between 0.2 and $1.1 \mathrm{~g}$. The device is designed to be comfortable and wearable, following the guidelines presented in [26]. Motors are encapsulated in a rigid core of dimensions $5 \times 20 \mathrm{~mm}$, and they are positioned evenly around the arm at 90 degrees from each other. They are connected to a small control board, which enables to control each motor independently. A Li-on battery ensures 4 hours of constant vibration at maximum intensity, and a Bluetooth Low Energy antenna enables wireless communication with an external control station. Fig. 1 shows one user driving the instrumented power wheelchair while wearing one vibrotactile armband, shown in Fig. 2.

Throughout the experiments, subjects were always able to look around and use their vision to drive the wheelchair, similarly to what most patients are able to do.

\section{EXPERIMENT \# 1:}

\section{USING A SINGLE VIBROTACTILE ARMBAND}

The objective of our first human subjects study is to evaluate the performance of using one vibrotactile armband for providing information either on a trajectory to follow or the presence of obstacles. The study has been approved by the UCL Research Ethics Committee (reference 6860/006). Following ethics requirements, all participants received a copy of the information sheet and signed a consent form.

\section{A. Setup}

Able-bodied participants were required to sit on our power wheelchair, with the vibrotactile armband fasten on their right upper arm, as shown in Figs. 1 and 3a. The circuit they had to navigate is shown in Fig. 4. It consisted of an S-shaped path starting with a corridor filled with obstacles. Then, it evolved into a larger space with a column in the middle. Finally, it ended in a small squared space having dimensions comparable to those of an elevator. The study took place at the Pedestrian Accessibility Movement Environment LAboratory (PAMELA), a facility at the University College of London (UCL) which allows full-scale pedestrian infrastructure to be built and tested.

\section{B. Experimental conditions}

We evaluate the effectiveness of two haptic feedback methods. The first one $\left(O_{w o z, 1}\right)$ provides users with information regarding the position of the closest obstacle; the second one $\left(G_{w o z, 1}\right)$ provides them 
with information on a target safe path to follow. In this first experiment, we used a Wizard-of-Oz approach. Participants perceived the feedback as being generated autonomously by the robotic wheelchair-armband system, when in reality the experimenter manually triggered the feedback when appropriate. This approach enabled the experimenter to easily and promptly switch the feedback strategy according to the live actions of the user, always conveying informative and appropriate guidance. Wizard-of-Oz techniques are common in the human-robot interaction design process [27], as they enable to test new ideas rather quickly and have an early feedback regarding what may or may not work (similarly to haptic sketching). The considered conditions are:

$O_{w o z, 1}:$ Motors vibrate to indicate the direction of the obstacle closest to the wheelchair, if said obstacle is closer than $1 \mathrm{~m}$. The feedback is discretized in 8 directions, as shown in Fig. $3 \mathrm{~b}$. Directions 2, 4, 6, 8 activate the motor in their circular sector at maximum intensity (e.g., direction 2 activates motor $\mathrm{A}$ at $280 \mathrm{~Hz} / 1.1 \mathrm{~g}$ ), while directions $1,3,5,7$ activate the two motors in their neighboring circular sectors at half intensity (e.g., direction 3 activate motors $\mathrm{A}$ and $\mathrm{B}$ at $180 \mathrm{~Hz} / 0.45 \mathrm{~g}$ ). Only information about the closest obstacle is provided with the bracelet.

$G_{w o z, 1}$ : Motors vibrate to indicate the direction to follow. We use the same discretization method described above (e.g., if a user should go right, toward direction 7 , we activate motors $\mathrm{C}$ and $\mathrm{D}$ at half intensity, $180 \mathrm{~Hz} / 0.45 \mathrm{~g}$ ).

$N_{1}$ : The armband is not active and the motors never vibrate.

Users were always able to see the surrounding environment while driving the wheelchair. All participants carried out the driving task in the three above conditions.

\section{Participants and task}

Nine healthy participants took part in this first experiment. The experimenter explained to each user, in detail, the conditions and the meaning of the feedback. Then, the experimenter adjusted the setup to be comfortable for the user. Participants were asked to sit on the wheelchair and wear one armband on their right upper arm. They were also allowed a 3-minutes practice driving session outside the circuit, to get familiar with the control of the wheelchair.

a) Preliminary perceptual test: Before the actual driving experiment, we had to ensure that participants could discriminate and well understand the eight considered directions. Hence, we carried out a preliminary perceptual test in which we explained them the feedback division and policy of the two conditions $O_{w o z, 1}$ and $G_{w o z, 1}$. Then, we generated 50 random directions per subject. For each direction, the vibrotactile armband provided the user with the corresponding vibrotactile guidance feedback as indicated before, and subjects had to tell the provided direction. Participants recognized the exact direction $44 \%$ of the times and $45 \%$ of the times they confused neighboring directions (e.g., 7 instead of 8), leading to an overall satisfactory recognition rate.

b) Driving experiment: After the preliminary perceptual test, participants were asked to enter the circuit, drive the wheelchair until its end, enter the elevator space, and come back. They were also asked to pay attention to the vibrotactile feedback provided by the armband, according to the conditions described in Sec. III-B. Figs. $4 \mathrm{c}$ and $4 \mathrm{~d}$ show a representative trajectory for one of the subjects. As the last part of the circuit was quite narrow, users were forced to proceed in reverse (red path) for around $5 \mathrm{~m}$ before driving again forward (blue path). The task starts when the human operator enters the circuit for the first time and finishes when he or she has completed a full lap. A video of this experiment is available as supplemental material and at https://youtu.be/NJRGXs28_wI.

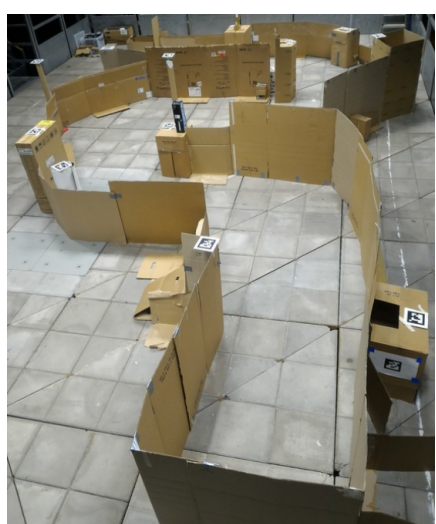

(a) Real picture of the circuit.

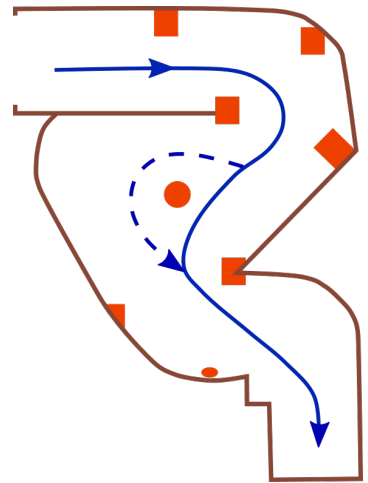

(c) First part of the task (driving always forward).

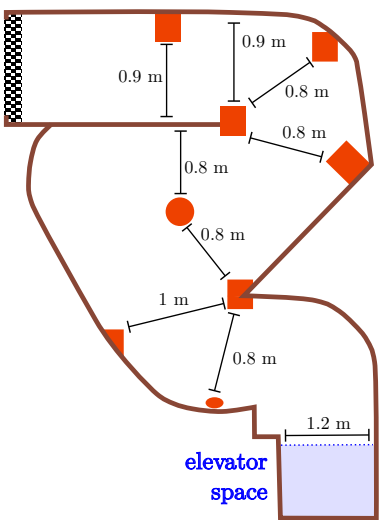

(b) Map of the circuit.

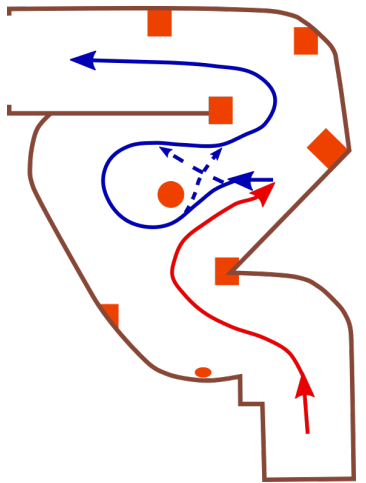

(d) Second part of the task (first backward, then forward).
Fig. 4. Experiment \#1: circuit. It is composed of an S-shaped structure filled with obstacles, ending in a narrow squared space resembling an elevator. Walls are depicted in brown, obstacles in orange, forward motion in blue, backward motion in red. Solid lines show a representative path taken by one of the subjects, dashed lines show alternative paths taken by other participants.

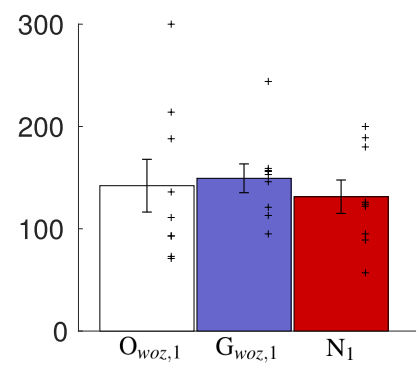

(a) Average completion time (s).

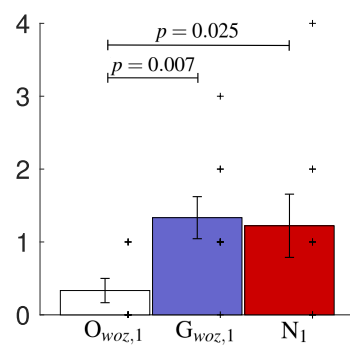

(b) Average number of collisions.
Fig. 5. Experiment \#1: results. Mean, 95\% confidence interval, and individual data points of the (a) average task completion time and (b) average number of collisions.

\section{Results and Discussion}

To evaluate the effectiveness of our system in navigating the circuit and the usefulness of wearable haptic feedback in such situations, we evaluated (i) the completion time and (ii) the number of collisions between the wheelchair and the environment. To compare the different metrics, we ran one-way repeated-measures inferential statistical tests on the data. The feedback condition $\left(O_{w o z, 1}\right.$ vs. $G_{w o z, 1}$ vs. $\left.N_{1}\right)$ is treated as the within-subject factor.

Figure 5a shows the average completion time. Data was transformed using the arcsin transformation. Transformed data were assessed to be 


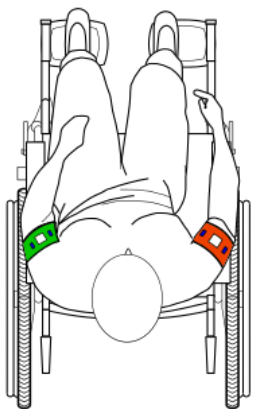

(a) Armbands positioned on both upper arms.

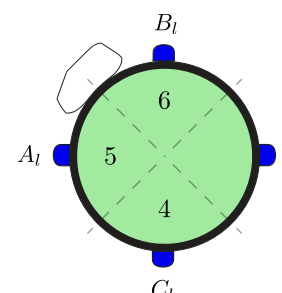

(b) Six discretized directions, provided by the joint action of six vibrotactile motors.
Fig. 6. Experiment \#2: setup. Users wear two vibrotactile armbands, on their right and left upper arms. The armband provides information either on a trajectory to follow or the presence of obstacles, in the form of a direction with respect to the current wheelchair orientation.

normally distributed and passed the Mauchly's Test of Sphericity. A one-way repeated-measure ANOVA revealed no statistically significant change in this metric $(F(2,16)=0.600, p>0.1, a=0.05)$. Figure $5 b$ shows the average number of collisions. A collision was counted when the wheelchair or the user came in contact with any obstacle or the wall. A Friedman test showed a statistically significant difference between the means of the four feedback conditions $\left(\chi^{2}(2)=10.786, p=0.005\right.$, $\mathrm{a}=0.05)$. The Friedman test is the non-parametric equivalent of the more popular repeated-measures ANOVA. The latter is not appropriate here since the dependent variable was measured at the ordinal level. Post hoc analysis with Bonferroni adjustments revealed a statistically significant difference between $O_{w o z, 1}$ vs. $N_{1}(p=0.025)$ and $O_{w o z, 1}$ vs. $G_{w o z, 1}(p=0.007)$.

Results show a significant decrease of the average number of collisions for condition $O_{w o z, 1}$ with respect to providing no haptic feedback. However, quite surprisingly, providing guidance feedback, $G_{w o z, 1}$, did not improve the safety of the driving task. Talking with the participants after the experiment, we understood that vibrating the motors toward the direction to follow became quickly uncomfortable. Users significantly preferred being notified only when an obstacle was close, instead of receiving constant information regarding the direction to follow. Indeed, for long-term use, it seems that providing constant vibrotactile feedback might lead the users to neglect the provided information. These comments are interesting but also somewhat surprising, as in our circuit users were often close to an obstacle, leading to the bracelet being active for around $70 \%$ of the time. Of course, in an open environment, where obstacles are further from each other, we expect the bracelet to be less active. We discuss in Sec. V the possibility of making the activation threshold tunable. Finally, a few participants reported that it was sometime confusing to receive all that information through a single bracelet, especially when the system told them to go left (the bracelet was worn on the right arm).

\section{EXPERIMENT \#2:}

\section{USING TWO VIBROTACTILE ARMBANDS}

Learning from the above results, the second human subjects experiment aims to evaluate the performance of using two wearable vibrotactile armbands in a driving task. The study has been approved by the UCL Research Ethics Committee (reference 6860/010). Following ethics requirements, all participants received a copy of the information sheet and signed a consent form.

\section{A. Setup}

Able-bodied participants were required to sit on our power wheelchair, with two vibrotactile armbands fasten on their right and

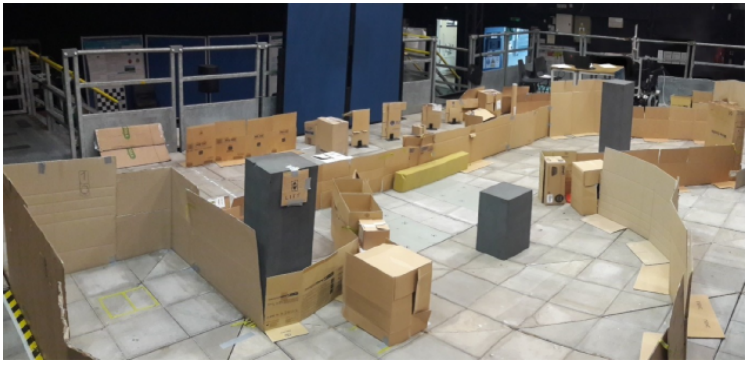

(a) Real picture of the circuit.

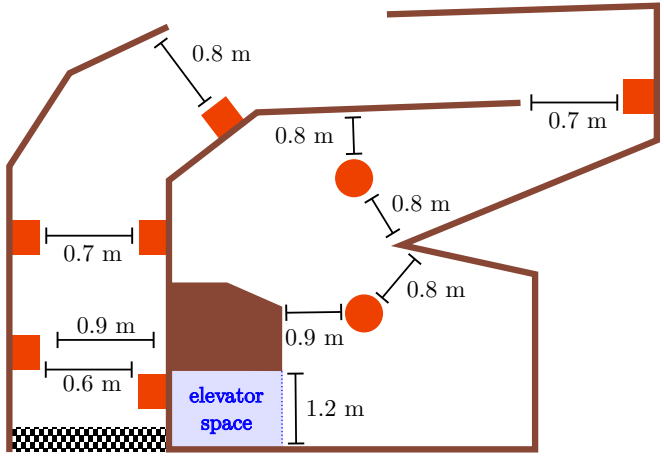

(b) Map of the circuit.

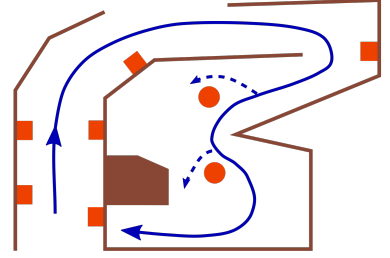

(c) First part of the task (driving always forward).

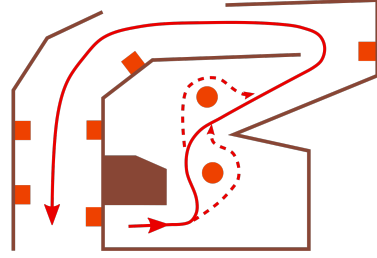

(d) Second part of the task (driving always backward).
Fig. 7. Experiment \#2: circuit. It is composed of a reversed U-shaped structure filled with obstacles, ending in a narrow squared space resembling an elevator Walls are depicted in brown, obstacles in orange, forward motion in blue, backward motion in red. Solid lines show a representative path taken by one of the subjects, dashed lines show alternative paths taken by other participants.

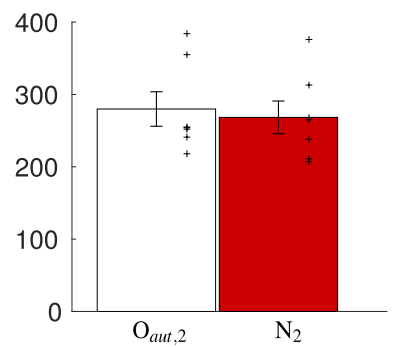

(a) Average completion time (s).

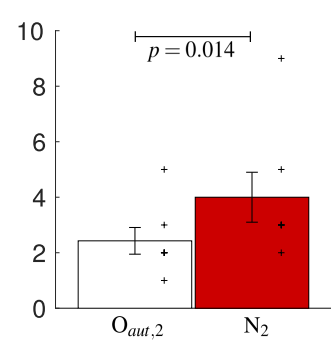

(b) Average number of collisions.
Fig. 8. Experiment \#2: results. Mean, 95\% confidence interval, and individual data points of the (a) average task completion time and (b) average number of collisions.

left upper arms, as shown in 6a. The circuit they had to navigate is shown in Fig. 7. It consisted of a reversed U-shaped path starting with a long corridor filled with obstacles. Then, it evolved into a larger space with two columns in the middle. Finally, it ended in a small squared space having dimensions comparable to those of an elevator The study took again place at the Pedestrian Accessibility Movement 
Environment LAboratory (PAMELA).

\section{B. Experimental conditions}

Given the good performance of $O_{w o z, 1}$, in this second experiment, we focused on the implementation and evaluation of a new condition providing information regarding the position of the closest obstacle Differently from before, we implemented an automatic recognition of the closest obstacle, using the set of ultrasonic sensors available on the wheelchair (see Fig. 1). The considered conditions are:

$O_{\text {aut }, 2}$ : Motors vibrate to indicate the direction of the obstacle closest to the wheelchair, if closer than $1 \mathrm{~m}$. The feedback is discretized in 6 directions across the two armband, as shown in Fig. 6. Each direction activates the motor in its circular sector at maximum intensity (e.g., direction 2 activates motor $A_{r}$ ). Only information about the closest obstacle is provided with the bracelet and only if said obstacle stays within the threshold for more than $200 \mathrm{~ms}$.

$N_{2}$ : The armbands are not active and the motors never vibrate. Users were always able to see the surrounding environment while driving the wheelchair. All participants carried out the driving task in the two above conditions.

\section{Participants and task}

Seven able-bodied participants took part in this second experiment They were different than those who participated to Experiment \#1. The experimenter explained to each user, in detail, the conditions and the meaning of the feedback. Then, the experimenter adjusted the setup to be comfortable for the user. Participants were asked to sit on the wheelchair and wear the armbands on their right and left upper arms. They were also allowed a 3-minutes practice driving session outside the circuit, to get familiar with the control of the wheelchair

Then, participants were asked to enter the circuit, drive the wheelchair until its end, enter the elevator space, and come back all in reverse. Before entering the elevator space, users had to press a button on the wall, resembling an elevator call button. Once inside, before moving back, they had to press another button on the wall, resembling a floor selection button. Participants were also asked to pay attention to the vibrotactile feedback provided by the armbands, according to the conditions described in Sec. IV-B. Figs. 7c and $7 \mathrm{~d}$ show a representative trajectory for one of the subjects. Users arrived to the elevator space driving forward (blue path), while they were asked to come back driving backward (red path). A video of this experiment is available as supplemental material and at https://youtu.be/NJRGXs28_wI.

\section{Results and Discussion}

We evaluated (i) the completion time, (ii) the number of collisions between the wheelchair and the environment, (iii) the usefulness of the vibrotactile feedback as perceived by the users, and (iv) the overall user's comfort in using the armbands. As before, we ran one-way repeated-measures inferential statistical tests on the data. The feedback condition $\left(\mathrm{O}_{\text {aut }, 2}\right.$ vs. $\left.\mathrm{N}_{2}\right)$ is treated as the within-subject factor.

Figure 8 a shows the average completion time. Data was transformed using the arcsin transformation. Transformed data were assessed to be normally distributed. The task starts when the human operator enters the circuit for the first time and finishes when he or she has completed a full lap. A paired-samples t-test revealed no statistically significant change in this metric $(t(6)=1.299, p>0.1, \mathrm{a}=0.05)$. Figure $8 \mathrm{~b}$ shows the average number of collisions. A collision was counted when the wheelchair or the user came in contact with any obstacle or the wall. A related-samples Wilcoxon signed rank test showed a statistically significant difference between the means of the two feedback conditions $(U=28, p=0.014$, a $=0.05)$. After the experiment, subjects were asked to report on their experience. They were asked to rate the usefulness of the vibrotactile feedback and the comfort of the armbands using bipolar Likert-type five-point scales. Results show that the vibrotactile information has been appreciated and found useful ( 3.9 out of 5), that the device has been very comfortable to wear and use (4.3 out of 5), and that users were satisfied with the overall driving and feedback system (4.0 out of 5 ). None of the participants reported significant troubles in locating the source of the vibration and understanding its meaning. Finally, users reported vibrations to be most useful when driving backward and when turning.

Results show again a significant decrease of the average number of collisions for condition $O_{\text {aut }, 2}$ with respect to providing no haptic feedback, showing once more that wearable vibrotactile feedback is a viable approach to achieve a safer driving of power wheelchairs. Moreover, the subjective questionnaires showed a general acceptance of the proposed method and a high comfort of our armbands. User's experience and comfort are very important, as target users would use the system every day.

\section{General Discussion AND CONCLUSIONS}

We proposed the use of wearable vibrotactile feedback to help people with disability safely drive power wheelchairs. We employ one or two vibrotactile armbands to provide drivers with information either on the presence of obstacles or on a safe path to follow. The need for such a navigation tool has become clear during our work within the EU-funded project "ADAPT", during which we worked next to expert therapists and people with motion impairing disabilities. Our objective and motivation is to enable a larger set of disabled people safely drive power wheelchairs, as being able to move autonomously is one of the most important tools of empowering and inclusion in our society. While other navigation aids have been presented in the past, to the best of our knowledge, this is the first system providing navigation assistance on a power wheelchair via a fullywearable tactile solution. Its characteristics overcome most of the limitations of currently-available kinesthetic approaches, opening new avenues for the field of assistance technology and wearable haptics. For example, using the proposed wearable solution, the driver receives rich navigation information, but he or she is still able to deviate from the suggested path whenever necessary. This feature is very important. While most of the available kinesthetic solutions literally take control of the wheelchair, totally or partially, our technique puts the human user at the center. Our armbands are also easy to adapt to different limbs and suitable for all those people who cannot safely use a force-feedback joystick.

We evaluated our approach in two-real world scenarios, enrolling fifteen able-bodied participants. Results show that, in both cases, providing information on closest obstacle position improved significantly the safety of the driving task (least number of collisions). Moreover, participants expressed a positive feedback on the use of vibrotactile sensations as well as on the comfort of the armbands. This result is important, as the system is developed to be used very often, i.e., every time that the patient moves in a complex environment. On the other hand, providing vibrotactile feedback toward an obstaclefree direction to follow was not successful. Subjects found sustained vibrations uncomfortable. A possible workaround to this issue is to provide vibrotactile guidance cues only if the current trajectory differs substantially from the target one. It is also important to highlight that we considered rather difficult tasks, comprising driving in reverse, turning, calling an elevator, and navigating very narrow spaces. Both circuits ended in a small elevator space, so as to simulate a complex everyday activity for our patients. In the second circuit, users were also asked to call the elevator and select a floor by pressing two buttons on the wall. We also noticed significant differences in the 
driving abilities of the enrolled subjects. Some of them were very natural in driving the wheelchair, while others showed a rather jerky motion. However, driving style did not seem to be affect by the haptic feedback. It is also important to highlight that users were always able to use their vision to navigate the environment, proving that haptic feedback is useful even when visual feedback is available, which is the most common case. Moreover, many patients in a wheelchair have significantly less mobility that the healthy users we enrolled in these experiments, limiting their vision capabilities and span. For example, our subjects often rotated their head to assess the distance from an obstacle (e.g., see https://youtu.be/NJRGXs28_wI?t=47). Certain patients would not be able to perform such movements, making haptic feedback even more relevant for them.

One drawback of this work is that we only enrolled able-bodied participants, while our approach is clearly targeted to people with disabilities. However, it is a requirement of our Research Ethics Committee to show the effectiveness, viability, and safety of any technique we propose first on healthy subjects. Only after, we can seek their approval for testing the technology with disabled people through clinical trials. For this reason, human subjects experiments enrolling people with disabilities are next in our research plan. Moreover, we would also like to test other types of wearable tactile sensations, including skin stretch and pressure. We have already started to work on developing armbands able to provide these type of stimuli [28], [29], [30], and it would be interesting to evaluate their effectiveness in this scenario. We also want to test the proposed technique in an outdoor environment and make the system's parameters (e.g., distance threshold) tunable, so as to better adapt to different scenarios, patients, and driving abilities. Another interesting avenue is considering other approaches for providing guidance information. While the methods proposed in Experiment \#1 were not particularly appreciated, less intrusive techniques (e.g., providing directions only at junctions or when requested) might perform better. Along this line of research, we will also study how to implement effective guidance techniques in an autonomous way using adaptive path-planning algorithms such as Rapidly-exploring Random Trees (RRTs) or Model Predictive Control (MPC). Finally, we will also study how the presence of haptic feedback as well as its strength/frequency affects the task and driving performance (e.g., jerkiness of the trajectory and commands). A system could use a stiff guidance approach (e.g., stronger haptic feedback) when it is operated by novices, while it could implement a soft guidance approach (e.g., weaker haptic feedback) when it is operated by experts. This flexible approach could be useful when teaching patients how to use our guidance system, employing different levels of autonomy according to the operator's experience.

\section{REFERENCES}

[1] P. Hardy, "Powered wheelchair mobility: An occupational performance evaluation perspective," Australian Occupational Therapy Journal, vol. 51, no. 1, pp. 34-42, 2004.

[2] A. Erdogan and B. D. Argall, "The effect of robotic wheelchair control paradigm and interface on user performance, effort and preference: an experimental assessment," Robotics and Autonomous Systems, vol. 94 pp. 282-297, 2017.

[3] V. K. Narayanan, A. Spalanzani, and M. Babel, "A semi-autonomous framework for human-aware and user intention driven wheelchair mobility assistance," in Proc. IEEE/RSJ Int. Conf. on Intelligent Robots and Systems (IROS), 2016, pp. 4700-4707.

[4] A. Hurst and J. Tobias, "Empowering individuals with do-it-yourself assistive technology," in Proc. ACM SIGACCESS conf. on Computers and accessibility, 2011, pp. 11-18.

[5] G. Meyerson and B. Dewettinck, "Effect of empowerment on employees performance," Advanced Research in Economic and Management Sciences, vol. 2, no. 1, pp. 40-46, 2012.

[6] M. F. Levin, H. Sveistrup, and S. Subramanian, "Feedback and virtual environments for motor learning and rehabilitation," Schedae, vol. 1, pp. 19-36, 2010.
[7] T. A. Salthouse, T. M. Atkinson, and D. E. Berish, "Executive functioning as a potential mediator of age-related cognitive decline in normal adults." Journal of experimental psychology: General, vol. 132, no. 4, p. 566, 2003.

[8] Y. Gaffary and A. Lécuyer, "The use of haptic and tactile information in the car to improve driving safety: A review of current technologies," Frontiers in ICT, vol. 5, p. 5, 2018.

[9] C. Ho, R. Gray, and C. Spence, "Reorienting driver attention with dynamic tactile cues," IEEE Trans. Haptics, vol. 7, no. 1, pp. 86-94, 2013.

[10] H. Tan, R. Gray, J. J. Young, and R. Taylor, "A haptic back display for attentional and directional cueing," 2003.

[11] I. Hussain, L. Meli, C. Pacchierotti, G. Salvietti, and D. Prattichizzo, "Vibrotactile haptic feedback for intuitive control of robotic extra fingers." in World Haptics, 2015, pp. 394-399.

[12] M. Pielot and S. Boll, "Tactile wayfinder: comparison of tactile waypoint navigation with commercial pedestrian navigation systems," in Pervasive computing, 2010, pp. 76-93.

[13] J. Lieberman and C. Breazeal, "TIKL: Development of a wearable vibrotactile feedback suit for improved human motor learning," IEEE Transactions on Robotics, vol. 23, no. 5, pp. 919-926, 2007.

[14] T. L. Baldi, S. Scheggi, M. Aggravi, and D. Prattichizzo, "Haptic guidance in dynamic environments using optimal reciprocal collision avoidance," IEEE Robotics and Automation Letters, vol. 3, no. 1, pp. 265-272, 2017.

[15] J. Bimbo, C. Pacchierotti, M. Aggravi, N. Tsagarakis, and D. Prattichizzo, "Teleoperation in cluttered environments using wearable haptic feedback," in Proc. IEEE/RSJ Int. Conf. on Intelligent Robots and Systems (IROS), 2017, pp. 3401-3408.

[16] S. M. Petermeijer, D. A. Abbink, M. Mulder, and J. C. de Winter, "The effect of haptic support systems on driver performance: A literature survey," IEEE Trans. Haptics, vol. 8, no. 4, pp. 467-479, 2015.

[17] L. Marchal-Crespo, J. Furumasu, and D. J. Reinkensmeyer, "A robotic wheelchair trainer: design overview and a feasibility study," Journal of neuroengineering and rehabilitation, vol. 7, no. 1, p. 40, 2010.

[18] X. Chen and S. K. Agrawal, "Assisting versus repelling force-feedback for human learning of a line following task," in Proc. IEEE RAS \& EMBS Int. Conf. on Biomedical Robotics and Biomechatronics (BioRob), 2012, pp. 344-349.

[19] A. Kucukyilmaz and Y. Demiris, "Learning shared control by demonstration for personalized wheelchair assistance," IEEE Trans. Haptics, vol. 11 , no. 3, pp. 431-442, 2018.

[20] E. B. Vander Poorten, E. Demeester, E. Reekmans, J. Philips, A. Hüntemann, and J. De Schutter, "Powered wheelchair navigation assistance through kinematically correct environmental haptic feedback," in Proc. IEEE Int. Conf. on Robotics and Automation (ICRA), 2012, pp. 3706-3712.

[21] L. Devigne, F. Pasteau, M. Babel, V. K. Narayanan, S. Guegan, and P. Gallien, "Design of a haptic guidance solution for assisted power wheelchair navigation," in Proc. IEEE Int. Conf. on Systems, Man, and Cybernetics (SMC), 2018, pp. 3231-3236.

[22] M. Sahnoun and G. Bourhis, "Haptic feedback to assist powered wheelchair piloting," Assoc. Adv. Model. Simul. Techn. Enterprises, vol. 67, pp. 53-63, 2006.

[23] C. Pacchierotti, Cutaneous haptic feedback in robotic teleoperation Springer, 2015

[24] H. U. Yoon, N. Anil Kumar, and P. Hur, "Synergistic effects on the elderly people's motor control by wearable skin-stretch device combined with haptic joystick," Frontiers in Neurorobotics, vol. 11, p. 31, 2017.

[25] R. OConnor and M. Smith, "Wheelchairs and special seating for neurological condition," 2008.

[26] C. Pacchierotti, S. Sinclair, M. Solazzi, A. Frisoli, V. Hayward, and D. Prattichizzo, "Wearable haptic systems for the fingertip and the hand: taxonomy, review, and perspectives," IEEE Trans. Haptics, vol. 10, no. 4 , pp. 580-600, 2017.

[27] H. Hüttenrauch, K. S. Eklundh, A. Green, and E. A. Topp, "Investigating spatial relationships in human-robot interaction," in Proc. IEEE/RSJ Int Conf. on Intelligent Robots and Systems (IROS), 2006, pp. 5052-5059.

[28] M. Aggravi, F. Pausé, P. Robuffo Giordano, and C. Pacchierotti, "Design and evaluation of a wearable haptic device for skin stretch, pressure, and vibrotactile stimuli," IEEE Robotics and Automation Letters, vol. 3, no. 3, pp. 2166-2173, 2018.

[29] F. Chinello, C. Pacchierotti, J. Bimbo, N. G. Tsagarakis, and D. Prattichizzo, "Design and evaluation of a wearable skin stretch device for haptic guidance," IEEE Robotics and Automation Letters, vol. 3, no. 1, pp. 524-531, 2017.

[30] C. Pacchierotti, D. Prattichizzo, and K. J. Kuchenbecker, "Displaying sensed tactile cues with a fingertip haptic device," IEEE Trans. Haptics, vol. 8, no. 4, pp. 384-396, 2015 